\title{
BIBECHANA
}

A Multidisciplinary Journal of Science, Technology and Mathematics

ISSN 2091-0762 (online)

Journal homepage: http://nepjol.info/index.php/BIBECHANA

\section{Physico-chemical parameters of Seepage stream at Kushaha area}

\author{
Damodar Thapa Chhetry ${ }^{1}$ and Joydeb Pal $^{2}$ \\ ${ }^{1}$ Department of zoology, Post Graduate Campus, Biratnagar \\ ${ }^{2}$ Department of Zoology, North Bengal University , India \\ Article history: Article history: Received 22 November, 2011; Accepted 17 December, 2011
}

\begin{abstract}
Physico-chemical parameters of the Seepage stream of Kushaha area were studied for two years from July, 2002-June, 2004, once in every month at regular intervals. The monthly data were pooled in seasonal value. The maximum air temperature was recorded in rainy season during first year study period (July, 2002 to June, 2003) but in the second year study period (July, 2003 to June 2004) it was maximum in summer season. Water temperature was maximum in summer and lowest in winter season. Transparency, $\mathrm{PH}$, dissolved oxygen, total alkalinity, total hardness, chloride were recorded maximum in winter season. Free carbondioxide, and biological oxygen demand was maximum in summer season. The minimum transparency, total alkalinity, total hardness and chloride were recorded in rainy season. Dissolved oxygen, and $\mathrm{PH}$, were minimum in summer season. The minimum carbondioxide and BOD were recorded in winter season. Air temperature, water temperature, free carbondioxide, biological oxygen demand showed positive and significant correlation with each other. Similarly, transparency, $\mathrm{PH}$, total alkalinity, total hardness, chloride, showed positive and significant correlation with each other. Dissolved oxygen showed positive and significant correlation with $\mathrm{pH}$, total alkalinity, total hardness and chloride.
\end{abstract}

Keywords: Physico- chemical parameters; seepage system

\section{Introduction}

The physico- chemical condition of a water body has a direct influence on the organisms. Seasonal fluctuations of various physico- chemical parameters have an important role in the distribution, periodicity, qualitative and quantitative composition of biota in aquatic ecosystem. The knowledge of all these parameters are essential for identifying the suitability and fertility of an aquatic ecosystem.

Several limnological studies have been done in the rivers and lakes of Nepal. Hickel [1], Lohaman et al. [2], Jones et.al [3], McEachern [4], Aryal and Lacoul [5], Ormerod et al. [6] have contributed on limnological work. 


\section{Study area}

The seepage stream is situated outside the Koshi Tappu Wildlife Reserve, east to the eastern embankment of Sapta Koshi river. It is a perennial water body flowing from Kushaha Village development committee (VDC) to south, Bhantabari area. The study site - Kushaha VDC area, lies between $26^{\circ} 37^{\prime} 10.6^{\prime \prime} \mathrm{N}$ latitude and $87^{\circ} 01^{\prime} 38.2^{\prime \prime} \mathrm{E}$ longitude (Fig. 1). The seepage stream at kushaha area has $125 \mathrm{~m}$ to $250 \mathrm{~m}$ wide marshes on its fringe. The depth of water of seepage stream varies in different seasons. It is somewhat polluted due to human encroachment but rich in biodiversity.

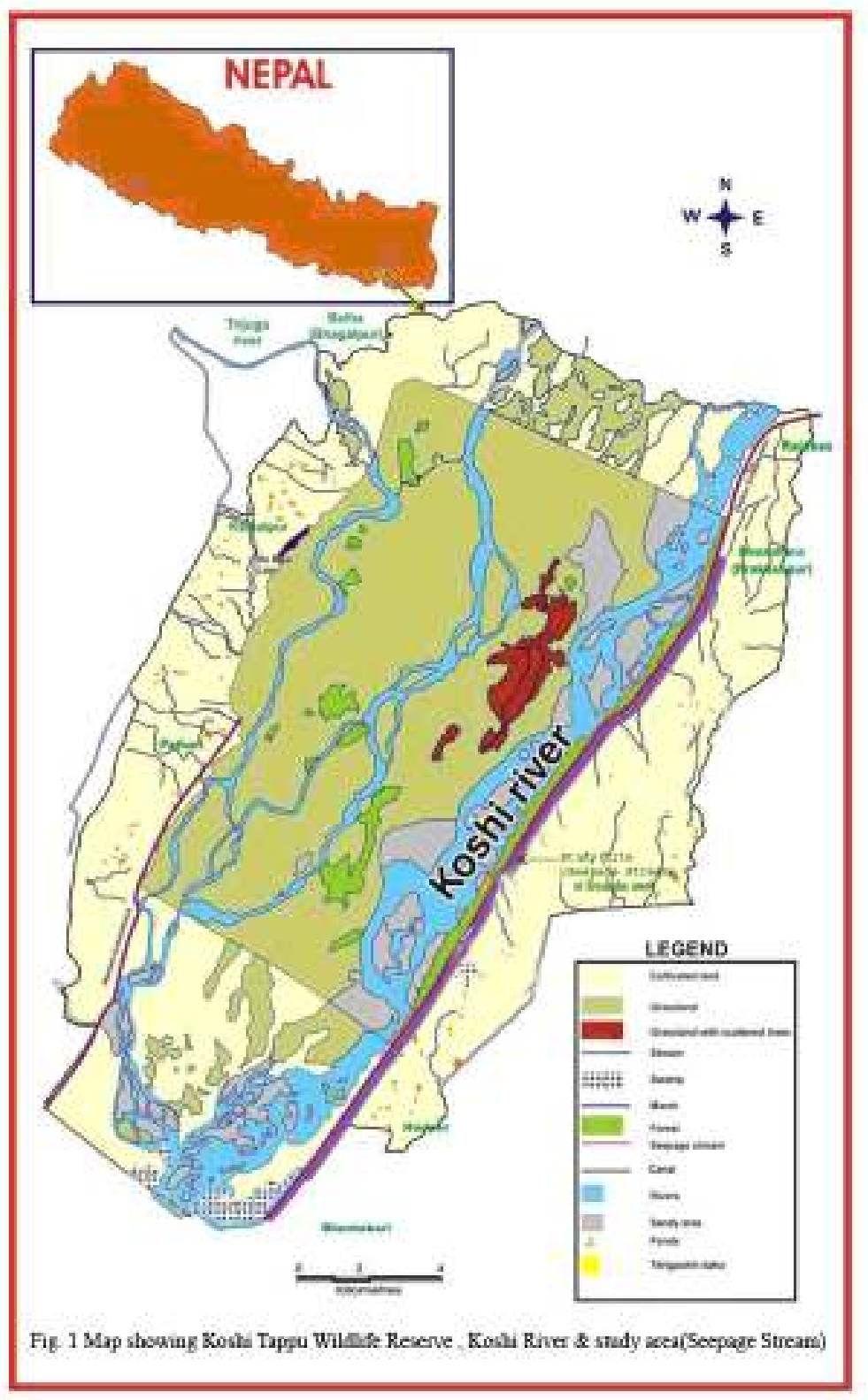


Damodar Thapa Chhetry and Joydeb Pal / BIBECHANA 8 (2012) 105-115 : BMHSS, p. 106

\section{Materials and Methods}

Physico-chemical parameters of the seepage stream were studied for two years from July, 2002June, 2004. The water samples were collected from three sites between 8 a.m. and 11 p.m., once in every month. The air temperature and physico- chemical parameters of water were analysed in the field. However, the BOD test after 5 days incubation in the incubator was done in the laboratory of Post Graduate Campus, Biratnagar. Transparency, air temperature and water temperature were recorded between 12 noon and 1 p.m. Air and water temperatures were recorded by centigrade mercury thermometer and $\mathrm{pH}$ with the help of $\mathrm{pH}$ meter. The transparency was measured by Secchi disc. Other parameters such as dissolved oxygen, free carbon dioxide, alkalinity, hardness, chloride, and BOD were measured according to Michael (7) Zobel et al. (8) and APHA (9).

\section{Results}

\subsection{Air temperature}

The minimum air temperature was $18.5 \pm 0.656{ }^{\circ} \mathrm{C}$ in the month of January and maximum $32.4 \pm$ $0.421{ }^{\circ} \mathrm{C}$ in August during the first year study period. The air temperature increased from July to August and decreased from September to January. Again it increased from February to May then a slight decrease was recorded in June during the first year study period (Table 1). During the second year study period, the air temperature increased a little in August then decreased from September to January. Again it increased from February to May then a slight decrease was recorded in June and July (Table 2). The maximum air temperature was $33.2 \pm 0.559{ }^{\circ} \mathrm{C}$ in May and minimum $19.3 \pm 0.382{ }^{\circ} \mathrm{C}$ in January during the second year study period. Air temperature showed positive and significant correlation with water temperature $(r=0.9794, P<0.01)$, free carbon dioxide $(r=0.6677, P<0.01)$ and biological oxygen demand $(r=0.6062, P<0.01)$ but it had inverse and significant correlation with transparency $(r=-0.452, \mathrm{P}<0.05), \mathrm{pH}(r=-0.908$, $P<0.01)$, dissolved oxygen $(r=-0.8994 P<0.01)$, total alkalinity $(r=-0.6713, P<0.01)$, total hardness $(r=-0.6239, P<0.01)$ and chloride $(r=-0.6759, P<0.01)$ (Table 3 ).

Seasonally, the highest air temperature was recorded in rainy season and minimum in winter season during the first year study period but it was highest in summer during the second year study period (Table 4).

\subsection{Water temperature}

The water temperature increased from July to August then decreased from September to January. Again it increased from February to May and a slight decrease was recorded in June during the first year (Table 1) and the second year study periods (Table 2). The maximum water temperature was recorded in May and minimum in January during the whole study period. The water temperature showed positive and significant correlation with free carbon dioxide $(r=$ $0.7319, P<0.01)$ and biological oxygen demand $(r=0.6960 P<0.01)$ but it showed inverse and significant correlation with transparency $(r=-3893, P<0.10), P^{H}(r=-0.917, P<0.01)$, dissolved oxygen $(r=-0.9229, P<0.01)$, total alkalinity $(r=-0.5613, P<0.01)$, total hardness $(r=-0.5167$, $P<0.01)$ and chloride $(r=-0.6645, P<0.01)$ (Table 3).

Seasonally, the maximum water temperature was in summer followed by rainy and winter seasons during the first and the second year study periods (Table 4). 
Damodar Thapa Chhetry and Joydeb Pal / BIBECHANA 8 (2012) 105-115 : BMHSS, p. 107

\subsection{Transparency}

The transparency increased from August to December then decreased a little in January. Again it increased from February to March then decreased a little in April and again increased a little in May then decreased from June to July during the first year study period (Table 1). The maximum

transparency was $37.34 \pm 0.650 \mathrm{~cm}$ in December and minimum $19.2 \pm 0.419 \mathrm{~cm}$ in July during the first year study period. In the second year study period, the transparency increased from August to December then decreased a little in January. Again it increased from February to March then decreased from April to July (Table 2). The maximum transparency was $33.2 \pm 1.001$ $\mathrm{cm}$ in March and minimum $20.2 \pm 0.986 \mathrm{~cm}$ in July during the second year study period. Water transparency showed positive and significant correlation with $\mathrm{pH}(\mathrm{r}=0.5196, \mathrm{P}<0.01)$, total alkalinity $(r=0.5621, P<0.01)$, total hardness $(r=0.8032, P<0.01)$ and chloride $(r=0.4667$, $\mathrm{P}<0.05)$ (Table 3).

The maximum value of transparency was in winter season followed by summer and rainy seasons during the first and second year study periods (Table 4).

\section{$3.4 \mathrm{pH}$}

The $\mathrm{pH}$ increased from July to December then decreased from January to May. Again it increased a little in June during the first year study period (Table 1). The maximum $\mathrm{pH}$ was $8.3 \pm 0.221$ in December and minimum $7.2 \pm 0.211$ in May during the first year study period. During the second year study period, $\mathrm{pH}$ increased from July to January then decreased from February to April. Again a slight increase was recorded in May then decreased a little in June (Table 2). The maximum pH was $8.3 \pm 0.205$ in January and minimum $7.1 \mathrm{mg} / \mathrm{l} \pm 0.163$ and $7.1 \pm$ 0.240 in April and June, respectively. $\mathrm{pH}$ showed positive and significant correlation with dissolved oxygen $(r=0.887, P<0.01)$, total alkalinity $(r=0.5583, P<0.01)$ total hardness $(r=$ $0.6099, P<0.01)$ and chloride $(r=0.6314, P<0.01)$ but inverse and significant correlation with free carbon dioxide $(r=-0.712, P<0.01)$ and biological oxygen demand $(r=-0.67, P<0.01)$ (Table 3).

The maximum $\mathrm{pH}$ was in winter and minimum in summer season during the first and the second year study period (Table 4).

\subsection{Dissolved oxygen}

The dissolved oxygen decreased from July to September then increased from October to January. Again it decreased from February to May and a slight increase was recorded in June during the first year study period (Table 1). The maximum dissolved oxygen was $6.96 \pm 0.268$ $\mathrm{mgl}$ in January and minimum $5.73 \pm 0.287 \mathrm{mgl}$ in May during the first year study period. During the second year study period, the dissolved oxygen decreased from July to August then increased from September to January. Again it decreased from February to May and a slight increase was recorded in June (Table 2). The maximum dissolved oxygen was $7.3 \pm 0.301 \mathrm{mg} / \mathrm{l}$ in January and minimum $5.92 \pm 0.219 \mathrm{mg} / \mathrm{l}$ in May. The dissolved oxygen showed positive and significant correlation with total alkalinity $(r=0.5241, P<0.01)$, total hardness $(r=0.4430$, $P<0.05)$ and with chloride $(r=0.6643, P<0.01)$ but inverse and significant correlation with free carbon dioxide $(r=-0.7342, P<0.01)$ and biological oxygen demand $(r=-0.7237, P<0.0 .01)$ (Table 3).

The maximum dissolved oxygen was in winter season followed by rainy and summer seasons during the first and the second year study periods (Table 4). 
Damodar Thapa Chhetry and Joydeb Pal / BIBECHANA 8 (2012) 105-115 : BMHSS, p. 108

Table 1 Air temperature and physico -chemical parameters of water of Seepage stream at Kuhasha area from July 2002 - June 2003 (Mean \pm S.D., N = 9).

\begin{tabular}{|c|c|c|c|c|c|c|c|c|c|c|c|c|}
\hline \multirow{2}{*}{ Parameters } & \multicolumn{12}{|c|}{ Months } \\
\hline & Jul. & Aug. & Sep. & Oct. & Nov. & Dec. & Jan. & Feb. & March & Apr. & May & Jun. \\
\hline \multirow[t]{2}{*}{$\begin{array}{c}\text { Air } \\
\text { temperature } \\
\left({ }^{\circ} \mathrm{C}\right)\end{array}$} & 31.3 & 32.4 & 28.3 & 27.6 & 22.7 & 21.2 & 18.5 & 27.6 & 29.4 & 30.3 & 31.3 & 29.2 \\
\hline & \pm 0.487 & \pm 0.421 & \pm 0.483 & \pm 0.609 & \pm 0.394 & \pm 0.397 & \pm 0.656 & \pm 0.586 & \pm 0.459 & \pm 0.385 & \pm 0.501 & \pm 0.294 \\
\hline \multirow{2}{*}{$\begin{array}{c}\text { Water } \\
\text { temperature } \\
\left({ }^{\circ} \mathrm{C}\right)\end{array}$} & 27.3 & 28.2 & 24.2 & 23.3 & 19.2 & 18.1 & 16.2 & 25.4 & 27.4 & 28.3 & 29.3 & 25.3 \\
\hline & \pm 0.485 & \pm 0.283 & \pm 0.427 & \pm 0.616 & \pm 0.326 & \pm 0.569 & \pm 0.343 & \pm 0.416 & \pm 0.639 & \pm 0.464 & \pm 0.589 & \pm 0.383 \\
\hline \multirow[t]{2}{*}{$\begin{array}{c}\text { Transparency } \\
\text { (cm). }\end{array}$} & 19.2 & 22.3 & 24.2 & 25.4 & 31.6 & 37.34 & 25.3 & 31.5 & 34.3 & 22.7 & 23.4 & 22.3 \\
\hline & \pm 0.419 & \pm 0.604 & \pm 0.721 & \pm 0.678 & \pm 0.548 & \pm 0.650 & \pm 0.512 & \pm 0.863 & \pm 0.818 & \pm 0.467 & \pm 0.705 & \pm 0.803 \\
\hline \multirow[t]{2}{*}{$\mathrm{PH}$} & 7.4 & 7.5 & 7.5 & 7.7 & 7.9 & 8.3 & 8.1 & 7.7 & 7.4 & 7.3 & 7.2 & 7.3 \\
\hline & \pm 0.176 & \pm 0.182 & \pm 0.216 & \pm 0.210 & \pm 0.194 & \pm 0.221 & \pm 0.188 & \pm 0.156 & \pm 0.216 & \pm 0.149 & \pm 0.211 & \pm 0.176 \\
\hline \multirow{2}{*}{$\begin{array}{c}\text { Dissolved } \\
\text { oxygen } \\
\left(\mathrm{DO}_{2}\right),(\mathrm{mg} / \mathrm{l})\end{array}$} & 6.18 & 6.15 & 6.13 & 6.29 & 6.61 & 6.91 & 6.96 & 6.26 & 5.98 & 5.92 & 5.73 & 6.25 \\
\hline & \pm 0.236 & \pm 0.216 & \pm 0.231 & \pm 0.222 & \pm 0.223 & \pm 0.277 & \pm 0.268 & \pm 0.165 & \pm 0.260 & \pm 0.253 & \pm 0.287 & \pm 0.205 \\
\hline \multirow{2}{*}{$\begin{array}{l}\text { Free carbon } \\
\text { dioxide } \\
\left(\mathrm{CO}_{2}\right),(\mathrm{mg} / \mathrm{l})\end{array}$} & 5.12 & 5.63 & 5.75 & 5.33 & 4.92 & 4.83 & 5.23 & 5.46 & 5.79 & 5.84 & 5.75 & 5.35 \\
\hline & \pm 0.215 & \pm 0.221 & \pm 0.222 & \pm 0.156 & \pm 0.154 & \pm 0.211 & \pm 0.188 & \pm 0.240 & \pm 0.214 & \pm 0.236 & \pm 0.170 & \pm 0.221 \\
\hline \multirow{2}{*}{$\begin{array}{c}\text { Total } \\
\text { alkalinity (mg/ } \\
\text { I) }\end{array}$} & 71.33 & 67.64 & 60.35 & 72.53 & 95.45 & 108.34 & 110.64 & 96.56 & 94.67 & 91.58 & 89.6 & 79.63 \\
\hline & \pm 0.725 & \pm 0.634 & \pm 0.683 & \pm 0.812 & \pm 1.023 & \pm 1.107 & \pm 1.040 & \pm 0.820 & \pm 0.728 & \pm 1.014 & \pm 0.805 & \pm 0.783 \\
\hline \multirow{2}{*}{$\begin{array}{c}\text { Total } \\
\text { hardness } \\
(\mathrm{mg} / \mathrm{l})\end{array}$} & 50.52 & 56.34 & 61.25 & 63.52 & 75.56 & 76.33 & 78.65 & 79.34 & 81.22 & 63.65 & 53.34 & 52.56 \\
\hline & \pm 0.539 & \pm 0.389 & \pm 0.437 & \pm 0.475 & \pm 0.405 & \pm 0.349 & \pm 0.391 & \pm 0.368 & \pm 0.535 & \pm 0.321 & \pm 0.585 & \pm 0.435 \\
\hline \multirow[t]{2}{*}{$\begin{array}{l}\text { Chloride (mg/ } \\
\text { I) }\end{array}$} & 13.24 & 13.83 & 14.23 & 14.45 & 15.26 & 18.73 & 17.27 & 16.36 & 14.84 & 13.14 & 14.45 & 17.23 \\
\hline & \pm 0.142 & \pm 0.156 & \pm 0.149 & \pm 0.163 & \pm 0.169 & \pm 0.205 & \pm 0.196 & \pm 0.157 & \pm 0.171 & \pm 0.134 & \pm 0.188 & \pm 0.216 \\
\hline \multirow{2}{*}{$\begin{array}{c}\text { Biological } \\
\text { Oxygen } \\
\text { Demand } \\
\text { (BOD), (mg/ } \\
\text { l) }\end{array}$} & 0.99 & 0.98 & 0.93 & 0.91 & 0.87 & 0.82 & 0.79 & 1.14 & 1.61 & 1.81 & 2.11 & 1.13 \\
\hline & \pm 0.033 & \pm 0.036 & \pm 0.049 & \pm 0.041 & \pm 0.045 & \pm 0.056 & \pm 0.052 & \pm 0.058 & \pm 0.075 & \pm 0.030 & \pm 0.055 & \pm 0.057 \\
\hline
\end{tabular}


Damodar Thapa Chhetry and Joydeb Pal / BIBECHANA 8 (2012) 105-115 : BMHSS, p. 109

Table 2 Air temperature and physico-chemical parameters of water of Seepage stream at Kuhasha area from July 2003 - June 2004 (Mean \pm S.D., $N=9$ ).

\begin{tabular}{|c|c|c|c|c|c|c|c|c|c|c|c|c|}
\hline \multirow{2}{*}{ Parameters } & \multicolumn{12}{|c|}{ Months } \\
\hline & Jul. & Aug. & Sep. & Oct. & Nov. & Dec. & Jan. & Feb. & March & Apr. & May & Jun. \\
\hline \multirow{2}{*}{$\begin{array}{c}\text { Air } \\
\text { temperature } \\
\left({ }^{\circ} \mathrm{C}\right)\end{array}$} & 30.3 & 31.4 & 29.3 & 28.3 & 23.5 & 22.4 & 19.3 & 28.4 & 30.3 & 31.4 & 33.2 & 30.3 \\
\hline & \pm 0.457 & \pm 0.416 & \pm 0.489 & \pm 0.410 & \pm 0.421 & \pm 0.402 & \pm 0.382 & \pm 0.571 & \pm 0.487 & \pm 0.405 & \pm 0.559 & \pm 0.485 \\
\hline \multirow{2}{*}{$\begin{array}{c}\text { Water } \\
\text { temperature } \\
\left({ }^{\circ} \mathrm{C}\right)\end{array}$} & 27.2 & 28.2 & 25.4 & 24.6 & 19.4 & 18.2 & 16.5 & 25.6 & 28.6 & 29.1 & 29.3 & 26.4 \\
\hline & \pm 0.346 & \pm 0.437 & \pm 0.346 & \pm 0.336 & \pm 0.480 & \pm 0.391 & \pm 0.249 & \pm 0.461 & \pm 0.336 & \pm 0.294 & \pm 0.489 & \pm 0.489 \\
\hline \multirow[t]{2}{*}{$\begin{array}{c}\text { Transparency } \\
\text { (cm). }\end{array}$} & 20.2 & 23.4 & 26.5 & 27.4 & 28.6 & 32.3 & 24.2 & 29.4 & 33.2 & 24.5 & 23.4 & 22.3 \\
\hline & \pm 0.986 & \pm 0.820 & \pm 0.890 & \pm 0.804 & \pm 0.909 & \pm 1.141 & \pm 0.653 & \pm 0.819 & \pm 1.001 & \pm 0.870 & \pm 0.857 & \pm 0.671 \\
\hline \multirow[t]{2}{*}{$\mathrm{PH}$} & 7.3 & 7.5 & 7.6 & 7.7 & 7.9 & 8.2 & 8.3 & 7.6 & 7.4 & 7.1 & 7.3 & 7.1 \\
\hline & \pm 0.176 & \pm 0.188 & \pm 0.163 & \pm 0.176 & \pm 0.176 & \pm 0.187 & \pm 0.205 & \pm 0.206 & \pm 0.230 & \pm 0.163 & \pm 0.226 & \pm 0.240 \\
\hline \multirow{2}{*}{$\begin{array}{c}\text { Dissolved } \\
\text { oxygen } \\
\left(\mathrm{DO}_{2}\right),(\mathrm{mg} / \\
\mathrm{l})\end{array}$} & 6.19 & 6.15 & 6.26 & 6.75 & 6.85 & 6.9 & 7.3 & 6.14 & 6.12 & 6.1 & 5.92 & 6.24 \\
\hline & \pm 0.224 & \pm 0.286 & \pm 0.254 & \pm 0.197 & \pm 0.156 & \pm 0.240 & \pm 0.301 & \pm 0.216 & \pm 0.239 & \pm 0.274 & \pm 0.219 & \pm 0.206 \\
\hline \multirow{2}{*}{$\begin{array}{l}\text { Free carbon } \\
\text { dioxide } \\
\left(\mathrm{CO}_{2}\right),(\mathrm{mg} / \mathrm{l})\end{array}$} & 5.23 & 5.57 & 5.92 & 5.14 & 5.1 & 4.73 & 5.23 & 5.9 & 6.2 & 5.93 & 5.89 & 5.87 \\
\hline & \pm 0.230 & \pm 0.257 & \pm 0.261 & \pm 0.216 & \pm 0.258 & \pm 0.258 & \pm 0.230 & \pm 0.210 & \pm 0.194 & \pm 0.235 & \pm 0.207 & \pm 0.240 \\
\hline \multirow{2}{*}{$\begin{array}{c}\text { Total } \\
\text { alkalinity (mg/ } \\
\text { l) }\end{array}$} & 69.45 & 66.78 & 61.52 & 74.52 & 98.72 & 107.83 & 111.73 & 98.64 & 94.57 & 93.52 & 87.65 & 77.76 \\
\hline & \pm 1.005 & \pm 0.931 & \pm 0.944 & \pm 0.929 & \pm 0.858 & \pm 1.003 & \pm 0.953 & \pm 0.916 & \pm 0.843 & \pm 1.098 & \pm 0.841 & \pm 0.810 \\
\hline \multirow{2}{*}{$\begin{array}{c}\text { Total } \\
\text { hardness } \\
(\mathrm{mg} / \mathrm{l})\end{array}$} & 51.45 & 54.35 & 60.65 & 63.52 & 65.34 & 69.89 & 77.65 & 78.34 & 82.49 & 56.34 & 54.11 & 53.54 \\
\hline & \pm 0.434 & \pm 0.307 & \pm 0.343 & \pm 0.415 & \pm 0.449 & \pm 0.373 & \pm 0.623 & \pm 0.408 & \pm 0.430 & \pm 0.655 & \pm 0.395 & \pm 0.279 \\
\hline \multirow[t]{2}{*}{$\begin{array}{l}\text { Chloride (mg/ } \\
\text { I) }\end{array}$} & 14.56 & 14.83 & 15.14 & 15.17 & 15.34 & 18.63 & 18.34 & 17.24 & 15.54 & 14.32 & 15.26 & 17.13 \\
\hline & \pm 0.221 & \pm 0.258 & \pm 0.206 & \pm 0.278 & \pm 0.283 & \pm 0.282 & \pm 0.236 & \pm 0.254 & \pm 0.240 & \pm 0.257 & \pm 0.233 & \pm 0.230 \\
\hline \multirow{2}{*}{$\begin{array}{c}\text { Biological } \\
\text { Oxygen } \\
\text { Demand } \\
\text { (BOD), (mg/ } \\
\text { l) }\end{array}$} & 1 & 0.98 & 0.89 & 0.85 & 0.84 & 0.78 & 0.72 & 1.1 & 1.56 & 1.79 & 2.14 & 1.1 \\
\hline & \pm 0.036 & \pm 0.048 & \pm 0.043 & \pm 0.042 & \pm 0.044 & \pm 0.048 & \pm 0.040 & \pm 0.042 & \pm 0.071 & \pm 0.055 & \pm 0.040 & \pm 0.048 \\
\hline
\end{tabular}


Damodar Thapa Chhetry and Joydeb Pal / BIBECHANA 8 (2012) 105-115 : BMHSS, p. 110

Table 3 Pearson's correlation coefficient $(r)$ for air temperature and physico - chemical parameters of water of Seepage stream at Kushaha area during July 2002 - June 2004. $\mathbf{N}=24$, d.f. $=22$.

\begin{tabular}{|c|c|c|c|c|c|c|c|c|c|}
\hline Parameters & $\begin{array}{c}\text { Water } \\
\text { temperatur } \\
\mathrm{e}\left({ }^{\circ} \mathrm{C}\right)\end{array}$ & $\begin{array}{c}\text { Transparenc } \\
\text { y }(\mathrm{cm}) .\end{array}$ & $\mathrm{P}^{\mathrm{H}}$ & $\begin{array}{c}\text { Dissolve } \\
\text { d oxygen } \\
\left(\mathrm{Do}_{2}\right) \\
(\mathrm{mg} / \mathrm{L})\end{array}$ & $\begin{array}{c}\text { Free } \\
\text { carbon } \\
\text { dioxide } \\
\left(\mathrm{Co}_{2}\right) \\
(\mathrm{mg} / \mathrm{L})\end{array}$ & $\begin{array}{c}\text { Total } \\
\text { alkalinit } \\
\text { y (mg/L) }\end{array}$ & $\begin{array}{c}\text { Total } \\
\text { hardnes } \\
\mathrm{s}(\mathrm{mg} / \mathrm{L})\end{array}$ & $\begin{array}{c}\text { Chloride } \\
\text { (mg/L) }\end{array}$ & $\begin{array}{c}\text { Biological } \\
\text { Oxygen } \\
\text { Demand } \\
(\mathrm{BOD}), \\
(\mathrm{mg} / \mathrm{L})\end{array}$ \\
\hline $\begin{array}{c}\text { Air } \\
\text { temperature } \\
\left({ }^{\circ} \mathrm{C}\right)\end{array}$ & $0.9794^{*}$ & $-0.452^{* *}$ & $-0.908^{*}$ & $-0.8994^{*}$ & $0.6677^{*}$ & $\stackrel{-}{-}^{-} 6713^{*}$ & $-0.6239^{*}$ & $-0.6759^{*}$ & $0.6062^{*}$ \\
\hline $\begin{array}{c}\text { Water } \\
\text { temperature } \\
\left({ }^{\circ} \mathrm{C}\right)\end{array}$ & & $-0.3893^{* * *}$ & $-0.917^{*}$ & $-0.9229^{*}$ & $0.7319^{*}$ & 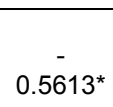 & $-0.5167^{*}$ & $-0.6645^{*}$ & $0.6960^{*}$ \\
\hline $\begin{array}{c}\text { Transparenc } \\
\text { y (cm). }\end{array}$ & & & $\underset{*}{0.5196}$ & 0.3216 & -0.1971 & $0.5621^{*}$ & $0.8032^{*}$ & $0.4667^{* *}$ & -0.1251 \\
\hline $\mathrm{P}^{\mathrm{H}}$ & & & & $0.887^{*}$ & $-0.712^{*}$ & $0.5583^{*}$ & $0.6099^{*}$ & $0.6314^{*}$ & $-0.67^{*}$ \\
\hline $\begin{array}{c}\text { Dissolved } \\
\text { oxygen } \\
\left(\mathrm{DO}_{2}\right),(\mathrm{mg} / \mathrm{l})\end{array}$ & & & & & $\stackrel{-}{0.7342^{*}}$ & $0.5241^{*}$ & $0.4430^{\star *}$ & $0.6643^{*}$ & $-0.7237^{*}$ \\
\hline $\begin{array}{l}\text { Free carbon } \\
\text { dioxide } \\
\left(\mathrm{CO}_{2}\right)(\mathrm{mg} / \mathrm{l})\end{array}$ & & & & & & -0.2834 & -0.1117 & $0.3789^{\star * *}$ & $0.6473^{*}$ \\
\hline $\begin{array}{c}\text { Total } \\
\text { alkalinity } \\
(\mathrm{mg} / \mathrm{L})\end{array}$ & & & & & & & $0.6943^{*}$ & $0.6284^{*}$ & 0.0548 \\
\hline $\begin{array}{c}\text { Total } \\
\text { hardness } \\
(\mathrm{mg} / \mathrm{l})\end{array}$ & & & & & & & & $0.4786^{* *}$ & -0.2052 \\
\hline $\begin{array}{c}\text { Chloride } \\
(\mathrm{mg} / \mathrm{l})\end{array}$ & & & & & & & & & $\begin{array}{c}- \\
0.3984^{* * *}\end{array}$ \\
\hline
\end{tabular}

(i) * Significant at $1 \%$ level $(P<0.01)$, ** significant at $5 \%$ level $(P<0.05)$, *** significant at $10 \%$ level $(P<0.10)$

(ii) Values not marked denote non-significant correlation.

Table 4 Seasonal variations in air temperature and physico-chemical parameters of water of Seepage stream at Kuhasha area during the first year (July 2002 - June 2003) and the second year (July 2003 - June 2004) study periods.

\begin{tabular}{|c|c|c|c|c|c|c|}
\hline \multirow{2}{*}{ Parameters } & \multicolumn{3}{|c|}{ Year I } & \multicolumn{3}{|c|}{ Year II } \\
\hline & Summer & Rainy & Winter & Summer & Rainy & Winter \\
\hline Air temperature $\left({ }^{\circ} \mathrm{C}\right)$ & 29.65 & 30.3 & 22.5 & 30.83 & 30.33 & 23.38 \\
\hline Water temperature $\left({ }^{\circ} \mathrm{C}\right)$ & 27.6 & 26.25 & 19.2 & 28.15 & 26.8 & 19.68 \\
\hline Transparency $(\mathrm{cm})$. & 27.98 & 22 & 29.91 & 27.63 & 23.1 & 28.13 \\
\hline $\mathrm{P}^{\mathrm{H}}$ & 7.4 & 7.43 & 8 & 7.35 & 7.38 & 8.03 \\
\hline Dissolved oxygen $\left(\mathrm{DO}_{2}\right),(\mathrm{mg} / \mathrm{l})$ & 5.97 & 6.18 & 6.69 & 6.07 & 6.21 & 6.95 \\
\hline Free carbon dioxide $\left(\mathrm{CO}_{2}\right)(\mathrm{mg} / \mathrm{l})$ & 5.71 & 5.46 & 5.08 & 5.98 & 5.65 & 5.05 \\
\hline Total alkalinity (mg/l) & 93.10 & 69.74 & 96.74 & 93.60 & 68.88 & 98.2 \\
\hline Total hardness $(\mathrm{mg} / \mathrm{l})$ & 69.39 & 55.17 & 73.52 & 67.82 & 55 & 69.1 \\
\hline Chloride (mg/l) & 14.70 & 14.63 & 16.43 & 15.59 & 15.42 & 16.87 \\
\hline Biological Oxygen Demand (BOD), (mg/l) & 1.67 & 1.01 & 0.85 & 1.65 & 0.99 & 0.80 \\
\hline
\end{tabular}


Damodar Thapa Chhetry and Joydeb Pal / BIBECHANA 8 (2012) 105-115 : BMHSS, p. 111

\subsection{Free carbon dioxide}

The free carbon dioxide increased a little from August to September then decreased from October to December. Again it increased from January to April then decreased from May to July during the first year study period (Table 1). The maximum free carbon dioxide was recorded 5.84 $\pm 0.236 \mathrm{mg} / \mathrm{l}$ in April and minimum $4.83 \pm 0.211 \mathrm{mg} / \mathrm{l}$ in December during the first year study period. During the second year study period, the free carbon dioxide increased from August to September then decreased from October to December. Again it increased from January to March then decreased from April to July (Table 2). The maximum free carbon dioxide was 6. $2 \pm 0.194$ $\mathrm{mg} / \mathrm{l}$ in March and minimum $4.73 \pm 0.258 \mathrm{mg} / \mathrm{l}$ in the month of December during the second year study period. Free carbon dioxide showed positive and significant correlation with biological oxygen demand $(r=0.6473, P<0.01)$ and inverse and significant correlation with chloride $(r=-$ $0.3789, \mathrm{P}<0.10)($ Table 3$)$.

The free carbon dioxide was maximum in summer season followed by rainy and winter seasons during the first and the second year study periods (Table 4).

\subsection{Total alkalinity}

The total Alkalinity decreased from July to September then increased from October to January. Again it decreased from February to June during the first year (Table 1) and the second year study periods (Table 2). The maximum total alkalinity was recorded $110.64 \pm 1.040 \mathrm{mgl}$ in January and minimum $60.35 \pm 0.683 \mathrm{mg} / \mathrm{l}$ in September during the first year study period. During the second year study period, the maximum total alkalinity was recorded $111.73 \pm 0.953 \mathrm{mg} / \mathrm{l}$ in January and minimum $61.52 \pm 0.944 \mathrm{mg} / \mathrm{l}$ in September. The total alkalinity showed positive and significant correlation with total hardness $(r=0.6943, P<0.01)$ and chloride $(r=0.6284, P<0.01)$ (Table 3).

The maximum value of total alkalinity was in winter season followed by summer and rainy seasons during the first and second year study periods (Table 4).

\subsection{Total hardness}

The total hardness increased from August to March then decreased from April to July during the first year (Table 1) and the second year study periods (Table 2). The maximum hardness was $81.22 \pm 0.535 \mathrm{mg} / \mathrm{l}$ in March and minimum $50.52 \pm 0.539 \mathrm{mg} / \mathrm{l}$ in July during the first year study period. During the second year study period, the maximum total hardness was $82.49 \pm 0.430$ $\mathrm{mg} / \mathrm{l}$ in March and minimum was $51.45 \pm 0.434 \mathrm{mg} / \mathrm{l}$ in July. Total hardness showed positive and significant correlation with chloride $(r=0.4786, P<0.05)$ (Table 3$)$.

The maximum value of total hardness was in winter season followed by summer and rainy seasons during the first and second year study periods (Table 4).

\subsection{Chloride}

The chloride increased from August to December then decreased from January to April. Again it increased from May to June then a slight decrease was recorded in July during the first year (Table 2) and the second year study periods (Table 2). The maximum chloride was $18.73 \pm$ $0.205 \mathrm{mgl}$ in December and minimum $13.14 \pm 0.134 \mathrm{mg} / \mathrm{l}$ in April during the first year study period. During the second year study period, the maximum chloride was $18.63 \pm 0.282 \mathrm{mgl}$ in December and minimum $14.32 \pm 0.257 \mathrm{mgl}$ in April. Chloride showed inverse and significant correlation with biological oxygen demand $(r=-0.3984, \mathrm{P}<0.10)$ (Table 3$)$. 
The maximum value of chloride was in winter season followed by summer and rainy seasons during the first and second year study periods (Table 4).

\subsection{Biological oxygen demand}

The biological oxygen demand decreased from July to January then increased from February to May. Again it decreased a little in June during the first year (Table 1) and the second year study periods (Table 2). The maximum biological oxygen demand was $2.11 \pm 0.055 \mathrm{mg} / \mathrm{l}$ in the month of May and minimum $0.79 \pm 0.052 \mathrm{mgl}$ in the month of January during the first year study period. During the second year study period, the maximum biological oxygen demand was 2.14 $\pm 0.040 \mathrm{mgl}$ in the month of May and minimum $0.72 \pm 0.040 \mathrm{mgl}$ in the month of January. Biological oxygen demand showed positive and significant correlation with air temperature $(r=0.6062$ $P<0.01)$, water temperature $(r=0.6960 P<0.01)$ and free carbon dioxide $(r=0.6473 P<0.01)$ but it had inverse and significant correlation with $P^{H}(r=-0.67, P<0.01)$, dissolved oxygen $(r=-$ $0.7237, P<0.01)$, and chloride $(r=-0.3984 P<0.10)$ (Table 3).

Seasonally, biological oxygen demand was maximum in summer season followed by rainy and winter seasons during the first and the second year study periods (Table 4).

\section{Discussion}

The rainy season showed highest air temperature in the first year study period because gradual increase in air temperature was noticed during summer months (February, March, April and May). Air temperature showed positive and significant correlation with water temperature. Chakaraborty et al. (10), Kant and Anand (11) and Rawat et al. (12) also obtained strong positive correlation between air and water temperatures.

Generally, water temperature is influenced by air temperature and intensity of solar radiation. It was highest in summer and lowest in winter. Highest value was recorded in summer might be due to high air temperature and greater light penetration. Though the high air temperature appeared in rainy season in the first year study period, a little lower water temperature was recorded at that time in comparison to that of summer season. It might be due to high turbidity, high volume of water and greater velocity of water in rainy season. The water temperature showed inverse and significant correlation with dissolved oxygen. Bose and Gorai (13) also reported inverse and significant correlation between water temperature and dissolved oxygen. Welch (14) and Munawar (15) have observed that shallower the water body more quickly it reacts to the change in the temperature.

The maximum secchi disc transparency was recorded in winter followed by summer and rainy seasons. The maximum transparency was in winter due to lesser amount of suspended organic and inorganic materials and absence of rain. Higher transparency during winter months was recorded by Singh (16), Rawat et al. (12), and Mishra et al. (17). In this study minimum transparency was recorded in rainy season might be due to more sand particles and colloidal soil carried by the rain water. Similar trends were observed by Singh (18), Rawat et al. (12), and Mishra et al. (19).

The maximum $\mathrm{pH}$ was in winter season may be attributed to algal blooms because Hutchinson et al. (20) and Roy (21) have shown that the higher $\mathrm{pH}$ is associated with the phytoplankton maxima. The minimum $\mathrm{pH}$ recorded in summer may be due to low photosynthesis. Several workers have reported low $\mathrm{pH}$ during the low photosynthesis due to the formation of carbonic acid (Hannan and Yong (22) Cabecadas and Brogueira (23) Bais et al.(24). 
The maximum dissolved oxygen found in winter season may be due to low temperature. Similar observation were made by Moitra and Bhattacharya (25). The minimum dissolved oxygen was found in summer due to high temperature, and higher microbial demand for oxygen in decomposition of suspended organic matter (Bhowmick and Singh (26) Dissolved oxygen content showed inverse and significant correlation with water temperature. Bose and Gorai (13) also reported similar result.

The maximum free carbon dioxide was recorded in summer, it may be due to high temperature, high rate of decomposition of organic matter, low volume of water etc. Michael (27) stated that the concentration of carbon dioxide is directly correlated with the amount and nature of biological activity in water. The minimum free carbon dioxide was found in winter season. Pahwa and Mehrotra (28), Ray et al. (29) also found minimum free carbon dioxide in winter season.

The maximum total alkalinity was found in winter season due to high $\mathrm{pH}$. Chakraborty et al. (10), Singh (16) and Mishra et al. (19) also reported maximum total alkalinity during winter. Jhingran (30) reported water bodies having total alkalinity from 40 to $90 \mathrm{mg} / \mathrm{l}$ as medium productive and above $90 \mathrm{mg} / \mathrm{l}$ as highly productive. This investigation showed that the study area is suitable for aquatic production. Total alkalinity showed positive and significant correlation with total hardness. Barat and Jha, (31) also reported similar result.

The maximum total hardness in winter season might be due to low volume of water and slow current of water. Similar results were obtained by Misra et al. (17). Patralekh (32) pointed minimum quantity in rainy season may be due to more dilution of water. Ruttner (33) also recorded similar relationship.

Maximum chloride recorded in winter season might be due to more contamination by organic matters. Klein (34) pointed out a direct relationship between amount of chloride and level of pollution.

The maximum BOD obtained in summer may be due to low volume of water and high content of organic matter whereas minimum obtained in winter may be due to low temperature and retarded microbial activity for the decomposition of organic matters. Similar observations were also made by Singh (18). Ray and Devid (35) opined that high BOD value indicates organic waste pollution. BOD showed inverse and significant correlation with dissolved oxygen. Ray and David (35) also reported similar relationship.

\section{References}

[1] B. Hickel, Limnological investigations in lakes of the Pokhara valley, Nepal. Hydrobiol. 58 (1973) $659-672$.

[2] K. Lohman, Jones J.R., M.F. Knowlton and D.B. Swar M.A. Pamperl and B.J. Brazos, Pre and post monsoon limnological characteristics of lakes in the Pokhara and Kathmandu valleys, Nepal. Verh. Internat. Verin. Limnol. 23 (1988) 558-565.

[3] J.R. Jones, M.F. Knowlton and D.B. Swar, Limnological reconnaissance of water bodies in central and southern Nepal. Hydrobiologia 184 (1989)171-189.

[4] P. McEachern, Limnology and the natural wetlands survey. In: Safeguarding Wetlands in Nepal (Eds.B. Bhandari,T.B.Shrestha and P. McEachern (1994) pp. 89-103. IUCN, Nepal.

[5] S. Aryal and P. Lacoul (1996) Water quality and diversity of diatoms in Punyamati River, Nepal. Ecoprint 3 (1996) 45-49.

[6] S.J. Ormerod, S.T. Buckton, P.A Brewin, A. Jenkins, R.C.Johnson, U. Juttner and A. Suren, Biodiversity, chemistry and structure in streams of the Nepalese Himalaya. In: Proc. Inter. Confer. Ecohydrol. High Moun. (Eds. Chalise S.R. and Khanal N.R.), (1996) pp. 1197-200. ICIMOD, Kathmandu. 
Damodar Thapa Chhetry and Joydeb Pal / BIBECHANA 8 (2012) 105-115 : BMHSS, p. 114

[7] P.Michael, Ecological Methods for Field and Laboratory Investigation. Tata Mc Graw- Hill Publishing Co. New Delhi. (1984)

[8] D.B. Zobel, P.K. Jha, M.J. Behan and U.K.R.Yadav, "A Practical Manual for Ecology". Ratna Book Distributors, Kathmandu (1987).

[9] APHA Standard Methods for Examination of Water and Waste Water. 20th Edition. Washington, USA. (1998)

[10] R. Chakaraborty, D.P.Roy and S.B. Singh, A quantitative study of the plankton and physicochemical conditions of the river Jamuna at Allahabad in 1954-55. Ind J. Fish. 6 (1959)186203.

[11] S. Kant and V.K. Anand, Interrelationship of phytoplankton and physical factors in Ansar lake, Jammu, India. J. Ecology 5 (1978)134-140.

[12] M.S. Rawat, C.P. Juyal, R.C. Sharma, Morphometry and physico- chemical profile of high altitude lake Deoria Tal of Garwal Himalaya. J .Freshwater Biol 7 (1995) 1-6.

[13] S.K. Bose and A.C. Gorai, Seasonal fluctuation of plankton in relation to physico-chemical parameters of a freshwater Tank of Dhanbad, India. J. Freshwater Biol. 5 (1993) 133-140.

[14] P.S. Welch, Limnological Methods, 2nd. Edition. MC. Graw Hill Book Co. New York (1952).

[15] M. Munawar, Limnological studies of fresh water ponds of Hyderabad, India - I. The Biotope. Hydrobiologia. 35 (1970)127- 162.

[16] H.P. Singh, Distribution and seasonal fluctuation of certain physico-chemical features in the Brahmaputra river. J. Assam Sci. Soc. 32 (1990) 64-69.

[17] A.P. Mishra, B.K.Bora, and M. Sharma, Limnological investigation of a freshwater tributary Assam, India. . J .Freshwater (Biol).11 (1999) 1-5.

[18] M. Singh, Impact of human activities on the physico-chemical conditions of two fish ponds at Patna, India. J. Freshwater Biol. 7 (1995)13- 17.

[19] A.P. Mishra, B.K Bora. and M. Sharma Investigations on the seasonal variation of certain Physico- chemical parameters of a Beel, Assam, India. J .Freshwater (Biol).10 (1998) 83-87.

[20] A.H., Hutchinson, S.C. Zucas and M. McPhail, Seasonal variation in the chemicals and physical properties of the waters of the strait of Georgia in relation to phytoplankton. Trans.Roy. Soc.Canada.3 (1929) 177-183.

[21] H. Roy, Plankton ecology of river Hooghly (West Bengal). Ecology. 36 (1955) 169- 744.

[22] H.H. Hannan and W.J. Yong, The influence of a deep storage reservoir on the physicochemical limnology of a central Taxas river. Hydrobiol. 44 (1974) 177- 207.

[23] G. Cabecadas and M.J. Brogueira, Primary production and pigments in three low alkalinity connected reservoirs receiving mine wastes. Hydrobiol. 144 (1987) 173- 182.

[24] V.S. Bais, N.C. Agrawal, and A.Tazeen, Comparative study on seasonal changes in phytoplankton community in the Sagar Lake and Military Engineering Lake (M.P.). J. Freshwater Biol. 7 (1995)19-25.

[25] S.K. Moitra and B.K.Bhattacharya Some hydrobiological factors affecting plankton production in fish pond at Kalyani W.B. India. Icthyologia 4 (1965) 8-12.

[26] B.N. Bhowmick and A.S. Singh, Effect of sewage on physico- chemical characteristics and bacterial population in river Ganga at Patna. Ind. J. Ecol. 12 (1985) 141-146.

[27] R.G.Michael, Seasonal trends in physico-chemical factors and plankton of a freshwater fish pond and their role in fish culture. Hydrobiol. 33 (1929) 144-160.

[28] D.V.Pahwa, and S.N. Mehrotra Observations on fluctuaions in abundance of plankton in relation to certain hydrobiological conditions of river Ganga. Proc. Nat. Acad. Sci. 36B 2 (1966) 57-189.

[29] P. Ray, S.B. Singh and K.L. Sehgal, A study on some aspects of the river Ganga and Jamuna at Allahabad (U.P.) in 1958-59. Proc. Nat. Acad. Sci. 36B (1966) 235-272.

[30] V.G. Jhingran, Fish and Fisheries of India, 3rd edition. Hindustan Publ. corp, New Delhi (1991)

[31] S. Barat and P. Jha, Changes in the water quality and total coliform bacterial load in a stretch of river Mahananda at Siliguri city ,West Bengal. Asian J. of Microbiol. Biotech. Env. Sc. 4 (2002) 571-575 
Damodar Thapa Chhetry and Joydeb Pal / BIBECHANA 8 (2012) 105-115 : BMHSS, p. 115

[32] L. N. Patralekh Comparative account of physico -chemical properties of three freshwater ecosystems. J .Freshwater Biol.6 (1994) 115-119.

[33] F. Ruttner, Fundamentals of Limnology. University of Toronto Press, Toronto (1953).

[34] L. .Klein Aspects of River Pollution. Butherworths Scientific Publication, London (1957).

[35] P. Ray and A. David, Effect of industrial wastes and sewage upon the chemical and biological composition and fisheries of the river Ganga at Kanpur, UP Environ. Health, 8(1966)307. 